\title{
JSBSim Library for Flight Dynamics Modelling of a mini-UAV
}

\author{
Tomáš Vogeltanz and Roman Jašek \\ Tomas Bata University in Zlin, Faculty of Applied Informatics \\ Department of Informatics and Artificial Intelligence \\ nám. T.G. Masaryka 5555, 76001 Zlín, CZECH REPUBLIC
}

\begin{abstract}
This paper presents using of JSBSim library for flight dynamics modelling of a mini-UAV (Unmanned Aerial Vehicle). The first part of the paper is about general information of UAVs and about the fundamentals of airplane flight mechanics, forces, moments, and the main components of typical aircraft. The main section briefly describes a flight dynamics model and summarizes the information about JSBSim library. Then, a way of using the library for the modelling of a mini-UAV is shown. A basic script for lifting and stabilization of the UAV has been developed and described. Finally, the results of JSBSim test are discussed.
\end{abstract}

Keywords: aircraft; airplane; flight dynamics model; JSBSim; modelling; simulation; UAV

PACS: 07.05.Tp

\section{INTRODUCTION}

Mini-UAVs are an inexpensive alternative to manned aircraft for a lot of applications including surveying, aerial reconnaissance, agriculture, environmental monitoring, and safety. The research community does not clearly define the term mini-UAV (or small-UAV). [1] [2] [3] [4] Instead of creation new definition about weight and proportions of the mini-UAV, we can simply say, a UAV is mini (or small) if the strength of an average man suffices for lifting and moving the UAV without any trouble. For the remainder of this paper, the term mini-UAV is implied.

UAVs can be classified into close range, short range and long range according to its areas of mission. Any UAV system depends on its mission and range. However, most UAV systems include: airframe and propulsion systems, control systems and sensors to fly the UAV, sensors to collect information, launch and recovery systems, data links to get collected information from the UAV and send commands to it, and a ground control station. [3] [4] [5]

Every UAV design, construction, implementation and test is unique and presents different challenges to engineers, pilots, and test team. UAVs promise greater precision, but the auto-pilot system which keeps the vehicle in the air and in control is critical to the success of UAV systems. In many cases, testing newly developed autopilot systems in a virtual environment is the only way to guarantee absolute safety. Additionally, the model allows better repeatability in testing. [1] [2]

\section{AIRPLANE FLIGHT MECHANICS}

In order to model an aircraft in any Flight Dynamic Model, the aircraft geometry, the lift, drag, and side forces, and the roll, pitch, and yaw moments acting on the aircraft must be understood. It is also important to know the aircraft control surfaces and their effects on the aircraft motion. [3]

The equations of motion are composed of translational (force) equations and rotational (moment) equations and are called the six degree of freedom (6DOF) equations of motion. The aircraft can move in three dimensions in space and can rotate about three axes. The origin of the coordinate system of the UAV is located at its center of gravity. [1] [2] [3] [4] [6] [7]

The forces of lift, weight, drag, thrust, and side force act along the axes, forcing the aircraft to move in the axes direction. The three moments, yaw, roll, and pitch force the aircraft to turn around the axes. X-axis is the roll axis, pointing forwards along the symmetry axis. Y-axis is the pitch axis, pointing rightwards from aircraft. Z-axis is the yaw axis, pointing downwards. [1] [2] [3] [4]

Figure 1 shows the main components of typical aircraft. The airframe consists of fuselage, wings, horizontal stabilizer, elevator, vertical stabilizer, and rudder. The elevator is used to control the pitch of the aircraft. The rudder

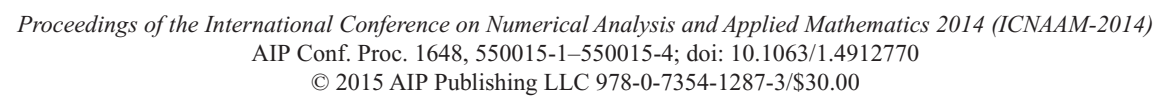


is used to control the yaw of the aircraft. The ailerons, which are movable surfaces on the outer trailing edge of the wings, are used to control the roll of the aircraft. The flaps, which are hinged parts on the inner trailing edge of the wings, are used to produce higher lift at low speed, and to increase drag on landing to get the required landing speed and approach angle. The landing gears configurations are either tricycle landing gear, which has the main landing gears just behind the aircraft center of gravity and a steerable nose gear, or tail dragger, which has the main landing gear forward of the aircraft center of gravity and a small steerable gear at the tail. [3]

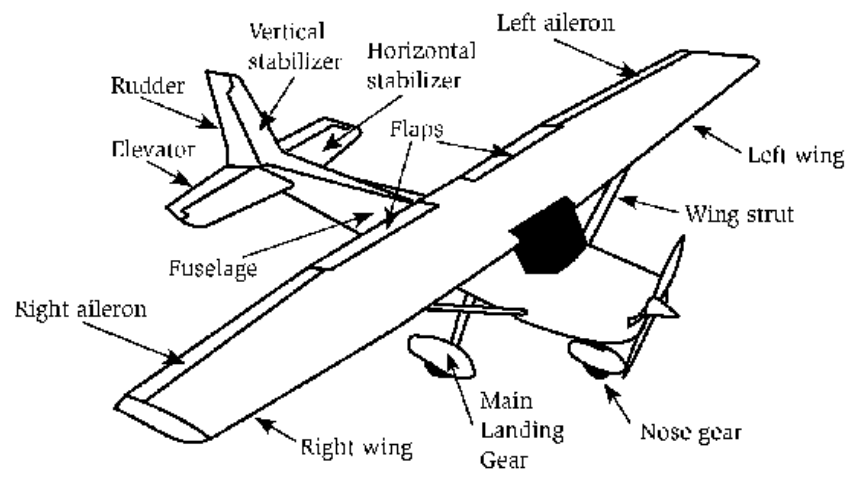

FIGURE 1. Typical aircraft components

\section{JSBSIM FLIGHT DYNAMICS MODEL}

Flight Dynamics Model (FDM) is the physics/math model which defines the movement of an aircraft under the forces and moments applied to it using the various control mechanisms and from the forces of nature. FDM includes development of a physical, inertial, and aerodynamic model representing the UAV. FDM processes parameters from all input information. By manipulating input variables mathematically, FDM predicts the future states of an aircraft. [1] [2] [3]

JSBSim is open source LGPL licensed software for a 6DOF nonlinear flight dynamics modelling. JSBSim is generally considered as a very accurate FDM and allows the modelling of any aircraft, missile, or rotorcraft, and models the rotational earth effects on the equation of motion. Particular aircraft flight control systems, propulsion, aerodynamics, landing gears, and autopilot are defined in eXtensible Markup Language (XML) format files. JSBSim supports many data output formats such as to screen, socket, and file. [1] [3] [8] [9]

\section{Aeromatic and Configuration Files}

The first step of the use of JSBSim to model and simulate a small autonomous UAV is to create the required JSBSim aircraft configuration files by using the Aeromatic. Aeromatic is a free web application and it is also included in source code of JSBSim. The next step is to make educated guesses to refine important sections in the created configuration files with the assistance of available data of similar UAV. [3] [9]

The aircraft's metrics, the aircraft's airframe geometry, mass and inertia properties, landing gear positions and their ground reactions, flight control system, and aerodynamic characteristics are specified in the aircraft configuration file. [3]

JSBSim can model different types of engines - electric, turbine, turboprop, piston, and rocket engines. The aircraft's propulsion system is specified in two files, one for the engine, and the other for the thruster. These files are referred to in the propulsion section in the main aircraft specification file which allows the researcher to assign different kinds of engines and thruster to the aircraft. [3] [9]

JSBSim can be scripted to make runs automatically. Commands are specified using the Scripting Directives for JSBSim. The script file is in XML format. A test condition (or conditions) can be set up in an event in a script and when the condition evaluates to true, the specified action(s) is/are taken. An event can be persistent, meaning that at all times when the test condition evaluates to true the specified set actions take place. When the set of tests evaluates to true for a given condition, an item may be set to another value. This value may be a value, or a delta value, and the change from the current value to the new value can be either via a step function, a ramp, or an exponential approach. The speed of a ramp or approach is specified via the time constant. [10] 


\section{JSBSim Take-off Test}

For the test, Rascal 110 UAV [11] has been used with one Zenoah G-26A Engine [12] and 18x8 propeller [13]. Parameters of the Rascal UAV are shown in Table 1. The engine power is $2207.27 \mathrm{~W}$. The propeller has 2 blades, rotational inertia of $0.00115 \mathrm{~kg}^{*} \mathrm{~m}^{2}$, minimal and maximal pitch angle of 30 inches/revolution.

TABLE 1. Parameters of the Rascal 110 UAV

\begin{tabular}{lc}
\hline Parameter & Value \\
\hline Wing Area $\left(\mathrm{m}^{2}\right)$ & 0.98 \\
Wing Span $(\mathrm{m})$ & 2.80 \\
Wing Chord $(\mathrm{m})$ & 0.35 \\
Horizontal Tail Area $\left(\mathrm{m}^{2}\right)$ & 0.16 \\
Horizontal Tail Arm $(\mathrm{m})$ & 1 \\
Vertical Tail Area $\left(\mathrm{m}^{2}\right)$ & 0.098 \\
Vertical Tail Arm $(\mathrm{m})$ & 0 \\
Empty Weight $(\mathrm{kg})$ & 5.9 \\
Payload $(\mathrm{kg})$ & 0.68 \\
\hline
\end{tabular}

The main goal of the created XML script is to lift the UAV, stop lifting after 5 seconds, and stabilize the UAV with using very primitive autopilot based on easy conditions.

The end of simulation was set to 30 seconds and initial altitude to 20 centimeters. After 0.1 second, the engine is started and the full power is set by using ramp function with time constant of 0.5 second.

Controlling and stabilization of the UAV flight was done by using elevator and ailerons only. After 5 seconds, a command to lift the UAV is sent. The lifting is stopped after next 5 seconds and then there is an attempt to stabilize the UAV. Figure 2 and Figure 3 show the process of velocity and altitude change.

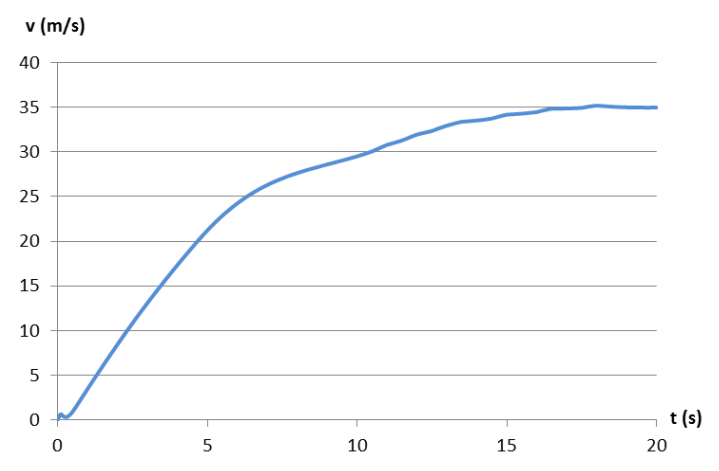

FIGURE 2. The process of velocity change of the UAV

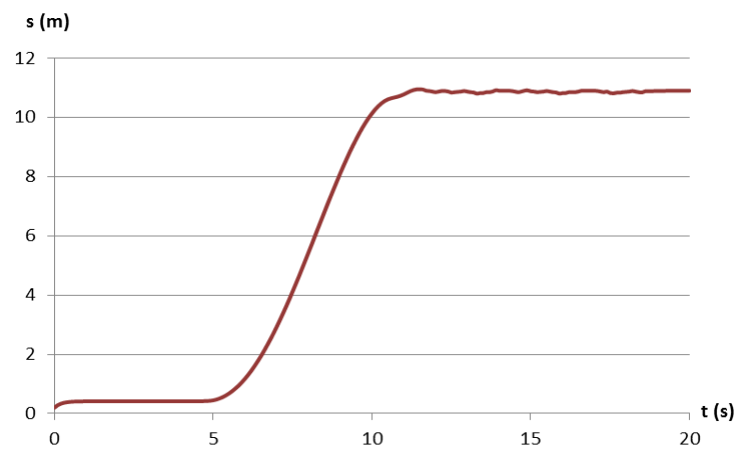

FIGURE 3. The process of altitude change of the UAV 
After engine start, the altitude of UAV is quickly stabilized at 40 centimeters without any input command. The UAV starts to lift off at the time of 5 seconds. Then, the UAV goes up by 10.16 meters during the 5.5 seconds. After a few tries, the UAV is completely stabilized at 10.9 meters. The UAV achieves top speed $(35 \mathrm{~m} / \mathrm{s}=126 \mathrm{~km} / \mathrm{h})$ at time of 18 seconds and it is stabilized almost at the same time.

\section{CONCLUSION}

This paper has presented using of JSBSim library for flight dynamics modelling of a mini-UAV. The flight dynamics model is the basis for the modelling of any aircraft. The JSBSim provides an excellent base for building the modelling environment. The fundamentals of airplane flight mechanics, the main components of typical aircraft, and flight dynamics model have also been mentioned.

The main section of this work has explained the JSBSim policy to flight dynamics modelling and has shown the options how to model aircraft, an engine, and a thruster and how to make and execute a script for testing of the created models.

The created script for lifting and stabilization of the modelled UAV has been described. Controlling and stabilization of the UAV flight was done by using elevator and ailerons only.

It has been found that the accurate stabilization of UAV with using primitive condition autopilot is difficult. It has been proved that when UAV soars, its velocity does not increase so fast and may decrease.

\section{ACKNOWLEDGMENTS}

This work was supported by the Internal Grant Agency at Tomas Bata University in Zlín, project no. IGA/FAI/2014/006.

\section{REFERENCES}

1. X.Q. Chen, Y.Q. Chen and J.G. Chase, Mobile Robots - State of the Art in Land, Sea, Air, and Collaborative Missions. Croatia: In-Teh, May 2009, pp.177-201.

2. N. M. Jodeh, Development of Autonomous Unmanned Aerial Vehicle. Ohio, USA: Wright-Patterson Air Force Base, March 2006, $185 \mathrm{p}$.

3. T. Abdunabi, Modelling and Autonomous Flight Simulation of a Small Unmanned Aerial Vehicle. Sheffield, UK: The University of Sheffield, August 2006, $61 \mathrm{p}$.

4. Z. Ye, P. Bhattacharya, H. Mohamadian, H. Majlesein, and Y. Ye, "Equational Dynamic Modeling and Adaptive Control of UAV," in Proceedings of the 2006 IEEE/SMC International Conference on System of Systems Engineering, Los Angeles, CA, USA, 2006, April, pp. 339-343.

5. R. Austin, Unmanned Air Systems: UAVs Design, Development and Deployment. Wiltshire, UK: Wiley, 2010,332 p.

6. D. G. Hull, Fundamentals of Airplane Flight Mechanics. Springer, 2007, 298 p.

7. T.V. Chelaru, V. Pana, A. Chelaru, "Dynamics and flight control of the UAV formations," WSEAS Transactions on Systems and Control, vol. 4 no. 4, pp. 198-210, April 2009.

8. JSBSim contributors. JSBSim Open Source Flight Dynamics Model. [Online]. Available: http://jsbsim.sourceforge.net/

9. JSBSim contributors. (2005, December 31). Aeromatic. [Online]. Available: http://jsbsim.sourceforge.net/aeromatic2.html

10. Jon S. Berndt and the JSBSim Development Team. (2011, June 9). JSBSim: An open source, platform-independent, flight dynamics model in $C++$. [Online]. Available: http://jsbsim.sourceforge.net/JSBSimReferenceManual.pdf

11. J. Goppert. (2013, January 13). Rascal.xml. [Online]. Available: https://github.com/PX4/HIL/blob/master/aircraft/Rascal/Rascal.xml

12. J. Goppert. (2013, January 13). Zenoah_G-26A.xml. [Online]. Available: https://github.com/PX4/HIL/blob/master/aircraft/Rascal/Engines/Zenoah_G-26A.xml

13. J. Goppert. (2013, January 13). 18x8.xml. [Online]. Available: https://github.com/PX4/HIL/blob/master/aircraft/Rascal/Engines/18x8.xml 
AIP Conference Proceedings is copyrighted by AIP Publishing LLC (AIP). Reuse of AIP content is subject to the terms at: http://scitation.aip.org/termsconditions. For more information, see http://publishing.aip.org/authors/rights-and-permissions. 